\title{
Four-center bubbled BPS solutions with a Gibbons-Hawking base
}

\author{
Pierre Heidmann \\ Institut de Physique Théorique, Université Paris Saclay, CEA, \\ Orme des Merisiers, F-91191 Gif-sur-Yvette, France \\ Département de Physique, École Normale Supérieure de Lyon, Université de Lyon, \\ 46 Allée d'Italie, F-69364 Lyon, cedex 07, France \\ E-mail: pierre.heidmann@cea.fr
}

\begin{abstract}
We construct four-center bubbled BPS solutions with a Gibbons-Hawking base space. We give a systematic procedure to build scaling solutions: starting from threesupertube configurations and using generalized spectral flows and gauge transformations to extend to solutions with four Gibbons-Hawking centers. This allows us to construct very large families of smooth horizonless solutions that have the same charges and angular momentum as supersymmetric black holes with a macroscopically large horizon area. Our construction reveals that all scaling solutions with four Gibbons Hawking centers have an angular momentum at around $99 \%$ of the cosmic censorship bound. We give both an analytical and a numerical explanation for this unexpected feature.
\end{abstract}

Keywords: Black Holes in String Theory, D-branes

ARXIV EPRINT: 1703.10095 


\section{Contents}

1 Introduction 1

2 Particular solutions with three BPS supertubes in Taub-NUT 3

2.1 Three-supertube BPS solutions in Taub-NUT 3

2.2 Bubble equations and regularity conditions 5

2.3 Examples of solutions 6

2.3.1 Specific choice of parameters and "scaling conditions" 6

2.3.2 Solutions with $h=0$ and with no constant terms in all $K^{I} \quad 7$

$\begin{array}{lll}2.3 .3 & \text { Solutions with } h \neq 0 & 8\end{array}$

3 Constructing BPS solutions with four Gibbons-Hawking centers $\quad 10$

$\begin{array}{lll}3.1 & \text { Four-GH center bubbled solutions } & 10\end{array}$

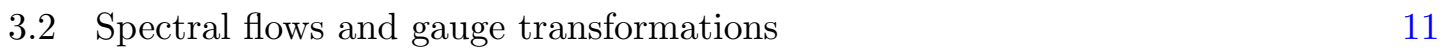

$\begin{array}{lll}3.3 & \text { One particular example of solutions } & 11\end{array}$

$\begin{array}{lll}3.3 .1 & \text { Scaling solutions } & 13\end{array}$

4 Discussion $\quad 14$

4.1 Solutions with four GH centers with no difference in scales are necessarily $\begin{array}{ll}\text { near-maximally spinning } & 14\end{array}$

$\begin{array}{lll}4.1 .1 & \text { Numerical analysis } & 14\end{array}$

$\begin{array}{lll}\text { 4.1.2 An analytical argument } & 15\end{array}$

$\begin{array}{llr}5 & \text { Conclusion } & 16\end{array}$

A Numerical analysis of the entropy parameter of four-GH center solutions 16

\section{Introduction}

Multi-center BPS bubbling solutions brought about important breakthroughs in the understanding of the microstate geometries of black holes. In the context of the fuzzball proposal (see [1-4] for reviews), they allow to understand the quantum structure of black holes by resolving singularities of classical black hole solutions into smooth, horizonless geometries. The main features of the geometries which replace the singularity are topologically nontrivial cycles called "bubbles" maintained by fluxes.

The physics of bubbled geometries is highly constrained by certain regularity conditions, known as the bubble equations, and the "no-CTC condition" $[5,6]$ which make the charges, the angular momenta and the positions of the centers all interdependent. Furthermore, to obtain horizonless microstate solutions with a black-hole-like throat, the bubbles 
need to "scale", that is to say, to shrink in the $\mathbb{R}^{3}$ base of the Gibbons-Hawking (GH) space $[7,8]$. Consequently, it is important to investigate the relation between charges, angular momenta and positions to understand the miscrostate geometries.

Bubbling BPS solutions with a Gibbons-Hawking base in five dimensions have been meticulously studied since a few years. A very large class of microstate geometries of supersymmetric black holes have been constructed from those solutions. The solutions can be written in a M2-M2-M2 frame or in other duality frames such as D1-D5-P or as D0-D4-F1 (for a review see [2]).

Finding specific examples of smooth horizonless multi-bubble solutions that have the same charges as a large black hole is quite non-trivial. Indeed, most solutions one can construct by putting fluxes on a multi-center Gibbons-Hawking base have angular momenta larger than the black hole cosmic censorship bound and hence can not be thought of as black hole microstate geometries. Trying to modify these fluxes to reduce the angular momenta gives very often rise to closed timelike curves. This is why in the literature, one can only find sparse specific examples of multi-center BPS solutions $[2,7,9,10]$. For example, one seven-center BPS three-charge black hole solution was built in [7]. The solution is scaling and the angular momentum $J$ is about $84 \%$ of its maximal value. However, some distances between the centers are 1000 times bigger than others.

In this article, we present a systematic construction and analysis of the largest known family of scaling four-center smooth horizonless solutions that have the same charges as large black holes. Our construction allows us to easily build scaling four-center BPS solutions with any aspect ratios between the centers. We study the charges and angular momentum of the solutions, as well as the entropy parameter, $\mathcal{H}$, given by

$$
\mathcal{H} \equiv \frac{Q_{1} Q_{2} Q_{3}-J^{2}}{Q_{1} Q_{2} Q_{3}} .
$$

The entropy parameter $\mathcal{H}$ indicates how far is the solution from its corresponding maximally spinning black hole solution. Our first surprising result is that all our solutions have a finetuned entropy parameter around 0.01. Several arguments lead us to the idea that four Gibbons-Hawking center BPS solutions which do not have an entropy parameter close to 0 are really rare. Moreover, we will show that when there is no difference in scales between the inter-center distances, all BPS solutions with four GH centers have an entropy parameter around 0,01 . This have been intuited before [11] and we give here new arguments and overall observations and we point out an important feature which is the difference in scale between the inter-center distances. Difference in scale should play a role in the value of the angular momentum and will be the subject of future work [12].

Furthermore, it is interesting to note that different directions have been found to construct microstate geometries with low angular momenta. Many recent works use superstratum technologies $[13,14]$ to generate microstate geometries with arbitrarily low angular momenta. This kind of microstates has the benefit of being parametrized by arbitrary continuous functions and not only harmonic functions so it seems to be a larger family of solutions. Furthermore, their CFT duals are exactly known unlike solutions with more than two Gibbons-Hawking centers. However, such microstate geometries still remain harder to 
construct than multicenter solutions. Moreover, the particular configurations with four GH centers under study in this paper are even easier to generate because they can be related by spectral flows to three supertubes in Taub-NUT (TN). Indeed, three-supertube constructions in Taub-NUT are easier to analyze than general four-GH center solutions and spectral flows are specific transformations which ensure that CTC are not introduced. Consequently, one can mostly concentrate on three-supertube constructions.

In section two, we construct several classes of specific solutions with three supertubes in TN. We give a systematic method to build examples of such scaling solutions. In section three, we use generalized spectral flow to construct bubbled BPS solutions with four GH centers from the three-supertube solutions. We then illustrate our method by constructing several classes of solutions with four GH centers. These solutions have an angular momentum $99 \%$ of the cosmic censorship bound. In section four, we present a numerical and an analytical proof that all solutions with four GH centers and black hole charges have such large angular momenta.

\section{Particular solutions with three BPS supertubes in Taub-NUT}

\subsection{Three-supertube BPS solutions in Taub-NUT}

The notations and the results are taken from [9, 15]. We will recall the three-charge solutions for a system of three supertubes in Taub-NUT. In M-theory, the three charges correspond to three M2 branes wrapping three orthogonal 2-tori inside a 6-torus. The eleven-dimensional metric is:

$$
d s_{11}^{2}=-\left(Z_{1} Z_{2} Z_{3}\right)^{-2 / 3}(d t+k)^{2}+\left(Z_{1} Z_{2} Z_{3}\right)^{1 / 3} d s_{4}^{2}+\left(Z_{1} Z_{2} Z_{3}\right)^{1 / 3} \sum_{I=1}^{3} \frac{d x_{I}^{2}+d y_{I}^{2}}{Z_{I}},
$$

where $d x_{I}^{2}+d y_{I}^{2}$ is the metric of the $\mathrm{I}^{\text {th }} 2$-Torus and $d s_{4}^{2}$ are the four-dimensional GibbonsHawking metric with one Gibbons-Hawking center:

$$
d s_{4}^{2}=V^{-1}(d \psi+A)^{2}+V\left(d x^{2}+d y^{2}+d z^{2}\right)
$$

with

$$
V=h+\frac{q}{r}, \quad \vec{\nabla} \times \vec{A}=\vec{\nabla} V
$$

The solutions have a three-form potential $\mathcal{A}$ :

$$
\mathcal{A}=\sum_{I=1}^{3} A^{(I)} \wedge d x_{I}^{2} \wedge d y_{I}^{2}
$$

The one forms $k$ and $A^{(I)}$ depend only on the four space coordinates transverse to the 6-Torus. The dipole field strengths $\Theta^{(I)}$ are defined as:

$$
\Theta^{(I)} \equiv d A^{(I)}+d\left(\frac{d t+k}{Z_{I}}\right)
$$


In order to have BPS solutions, the fields must satisfy the following equations $[16,17]$,

$$
\begin{aligned}
\Theta^{(I)} & =\star_{4} \Theta^{(I)} \\
\hat{\nabla}^{2} Z_{I} & =\frac{1}{2} C_{I J K} \star_{4}\left(\Theta^{(J)} \wedge \Theta^{(K)}\right) \\
d k+\star_{4} d k & =Z_{I} \Theta^{(I)} .
\end{aligned}
$$

The Hodge dual $\star_{4}$ is the Hodge dual of the four-dimensional Gibbons-Hawking space, and $C_{I J K}$ is the absolute value of the constant symmetric tensor.

We consider axisymmetric supertube configurations. The positions of the supertube centers are given by the distances $a_{1}, a_{2}$ and $a_{3}$ on the z-axis in the following order

$$
a_{1}>a_{2}>a_{3}>0 \text {. }
$$

The BPS equations (2.6) allow to consider three types of two-charge supertubes. Each supertube carries a dipole charge $k_{I}$ and two electric charges $Q_{j}^{(I)}$ at the points $j \neq I$. Consequently, the dipole strengths $\Theta^{(I)}$ and the scalar fields $Z_{I}$ are sourced by harmonic functions which we call $K^{I}$ and $L_{I}$

$$
\begin{aligned}
& K^{I}=\alpha_{I}+\frac{k_{I}}{r_{I}} \\
& L^{1}=1+\frac{Q_{2}^{(1)}}{4 r_{2}}+\frac{Q_{3}^{(1)}}{4 r_{3}} \quad, \quad L^{2} \text { and } L^{3} \text { by permutation, }
\end{aligned}
$$

where $r_{I}$ is the distance to the $\mathrm{I}^{\text {th }}$ center

$$
r_{I} \equiv \sqrt{x^{2}+y^{2}+\left(z-a_{I}\right)^{2}}
$$

The complete solution for the warp factors is:

$$
\begin{aligned}
Z_{I} & =L_{I}+\frac{1}{2} C_{I J K} \frac{K^{J} K^{K}}{V} \\
& =1+\sum_{J \neq I} \frac{Q_{J}^{(I)}}{4 r_{J}}+\frac{C_{I J K}}{h+\frac{q}{r}}\left(\frac{k_{J} k_{K}}{2 r_{J} r_{K}}+\frac{\alpha_{J} \alpha_{K}}{2}+\frac{\alpha_{J} k_{K}}{r_{K}}\right),
\end{aligned}
$$

and

$$
k=\mu(d \psi+A)+\omega,
$$

with

$$
\mu=\frac{1}{6} V^{-2} C_{I J K} K^{I} K^{J} K^{K}+\frac{1}{2} V^{-1} K^{I} L_{I}+M,
$$

where $\mathrm{M}$ is another harmonic function which we will take to be

$$
M=m_{\infty}+\frac{m_{0}}{r}+\sum_{j=1}^{3} \frac{m_{j}}{r_{j}},
$$

and $\omega$ is another vector which satisfies the following equation in the $\mathbb{R}^{3}$ base of $\mathrm{TN}$ :

$$
\vec{\nabla} \times \omega=V \vec{\nabla} \mu-\mu \vec{\nabla} V-V \sum_{I=1}^{3} Z_{I} \vec{\nabla}\left(\frac{K^{I}}{V}\right) .
$$


Thus, $\mu$ is

$$
\begin{aligned}
\mu= & \frac{1}{V^{2}}\left(\frac{k_{1} k_{2} k_{3}}{r_{1} r_{2} r_{3}}+\alpha_{1} \alpha_{2} \alpha_{3}+\frac{C_{I J K}}{2}\left(\frac{\alpha_{I} k_{J} k_{K}}{r_{J} r_{K}}+\frac{\alpha_{I} \alpha_{J} k_{K}}{r_{K}}\right)\right) \\
& +\frac{C_{I J K}}{4 V}\left(\alpha_{I}+\frac{k_{I}}{r_{I}}\right)\left(1+\frac{Q_{J}^{(I)}}{4 r_{J}}+\frac{Q_{K}^{(I)}}{4 r_{K}}\right)+M .
\end{aligned}
$$

\subsection{Bubble equations and regularity conditions}

In addition to the BPS equations (2.6), the solutions must satisfy several regularity conditions that we recall in this section. First, divergent terms along the $\psi$-fiber in the metric must vanish. Second, closed timelike curves (CTC) must be absent. We organize the harmonic functions in a symplectic vector $\mathrm{H}$ :

$$
\begin{aligned}
H & =\left(H^{0}, H^{I}, H_{I}, H_{0}\right) \equiv\left(V, K^{I}, L_{I}, 2 M\right) \\
& =\hat{h}+\sum_{j=0}^{3} \frac{\Gamma_{j}}{r_{j}}
\end{aligned}
$$

with

$$
\begin{aligned}
\hat{h} & =\left(h, \alpha_{1}, \alpha_{2}, \alpha_{3} ; 1,1,1,2 m_{\infty}\right), \quad \Gamma_{0}=\left(q, 0,0,0 ; 0,0,0,2 m_{0}\right), \\
\Gamma_{j} & =\left(0, k_{j} ; \frac{1}{4} Q_{j}, 2 m_{j}\right) .
\end{aligned}
$$

The conditions of regularity at the singularities $r_{j} \rightarrow 0 j=\{0,1,2,3\}$ with $r_{0}=r$ are summed up by the following equations [6]:

$$
\sum_{J=0}^{3} \frac{\left\langle\Gamma_{I}, \Gamma_{J}\right\rangle}{r_{I J}}=-\left\langle\Gamma_{I}, \hat{h}\right\rangle \quad I=\{0,1,2,3\},
$$

where $\langle$,$\rangle is a symplectic product defined as follows$

$$
\langle A, B\rangle=A_{0} B_{8}-A_{8} B_{0}+A_{I} B_{8-I}-A_{8-I} B_{I} .
$$

Using the notation

$$
\Gamma_{I J}=k_{I} Q_{J}^{(I)}-k_{J} Q_{I}^{(J)}
$$

we obtain the following bubble equations

$$
\begin{aligned}
& \frac{\Gamma_{12}}{r_{12}}+\frac{\Gamma_{13}}{r_{13}}=8 m_{1}\left(h+\frac{q}{a_{1}}\right)-4 k_{1}+Q_{1}^{(2)} \alpha_{2}+Q_{1}^{(3)} \alpha_{3} \\
& \frac{\Gamma_{21}}{r_{12}}+\frac{\Gamma_{23}}{r_{23}}=8 m_{2}\left(h+\frac{q}{a_{2}}\right)-4 k_{2}+Q_{2}^{(1)} \alpha_{1}+Q_{2}^{(3)} \alpha_{3} \\
& \frac{\Gamma_{32}}{r_{23}}+\frac{\Gamma_{31}}{r_{13}}=8 m_{3}\left(h+\frac{q}{a_{3}}\right)-4 k_{3}+Q_{3}^{(1)} \alpha_{1}+Q_{3}^{(2)} \alpha_{2} \\
& q\left(m_{\infty}+\sum_{j=1}^{3} \frac{m_{j}}{a_{j}}\right)=m_{0} h .
\end{aligned}
$$


Furthermore, all the parameters in $M$ are constrained to avoid Dirac strings at each pole and to avoid divergences along the $\psi$-fiber [15]:

$$
\begin{array}{ll}
m_{1}=\frac{Q_{1}^{(2)} Q_{1}^{(3)}}{32 k_{1}}, & m_{2}=\frac{Q_{2}^{(1)} Q_{2}^{(3)}}{32 k_{2}}, \quad m_{3}=\frac{Q_{3}^{(2)} Q_{3}^{(1)}}{32 k_{3}} \\
m_{0}=0, & m_{\infty}=-\sum_{j=1}^{3} \frac{m_{j}}{a_{j}}
\end{array}
$$

Finally, the absence of CTCs is satisfied when the quartic invariant $I_{4}$ is greater than $\omega^{2}$ in the three-dimensional base space of the solution [18]:

$$
I_{4} \equiv Z_{1} Z_{2} Z_{3} V-\mu^{2} V^{2}>\omega^{2} .
$$

\subsection{Examples of solutions}

The goal of this section is to construct several examples of supertube charges and dipole charges such that the bubble equations have physical solutions. In particular, we will focus on solutions with no difference in scales between the inter-center distances. We know from [7] that the depth of the solution's throat is inversely related to the distance between the centers in $\mathbb{R}^{3}$. Thus, the centers must cluster to form a concentrated object in order for the microstate geometry to look like a black hole when seen from large distances. Several methods exist for constructing such solutions. In general solutions scale when the centers move away from axisymmetry. However, to scale axisymmetric solutions we will slightly change the value of the charges, as in [7].

\subsubsection{Specific choice of parameters and "scaling conditions"}

We consider the following choice of charges and dipole charges inspired from the choice made in [9]

$$
\begin{aligned}
k_{1} & =k_{3}=-k_{2}=k>0 \\
Q_{1}^{(2)} & =Q_{3}^{(2)}=\alpha>0 \\
Q_{3}^{(1)} & =Q_{2}^{(3)}=\beta>0 \\
Q_{2}^{(1)} & =Q_{1}^{(3)}=\gamma>0 .
\end{aligned}
$$

Finding an explicit scaling condition is crucial. Indeed, one can find many solutions of the bubble equations which do not scale. However, one must be able to go to a scaling limit to build a solution which looks like a black hole from far away.

For this purpose, we solve the bubble equations (2.21) by computing the charges for a given cluster of supertubes infinitely close to the Gibbons Hawking center, that is to say in the limit $a_{i} \rightarrow 0$. So we consider an arbitrary arrangement of distances

$$
a_{j}=\lambda d_{j} \quad j=\{1,2,3\}
$$

with

$$
\lambda \ll 1, \quad d_{1}>d_{2}>d_{3} .
$$


We linearize the bubble equation (2.21) in powers of $\lambda$

$$
\begin{aligned}
& \frac{1}{\lambda}\left(\frac{\alpha+\gamma}{d_{1}-d_{2}}+\frac{\beta-\gamma}{d_{1}-d_{3}}-\frac{q}{4 k^{2} d_{1}} \alpha \gamma\right)=\frac{h}{4 k^{2}} \alpha \gamma-4+\frac{\alpha \alpha_{2}+\gamma \alpha_{3}}{k} \\
& \frac{1}{\lambda}\left(-\frac{\alpha+\gamma}{d_{1}-d_{2}}-\frac{\alpha+\beta}{d_{2}-d_{3}}+\frac{q}{4 k^{2} d_{2}} \beta \gamma\right)=-\frac{h}{4 k^{2}} \alpha \gamma+4+\frac{\gamma \alpha_{1}+\beta \alpha_{3}}{k} \\
& \frac{1}{\lambda}\left(\frac{\gamma-\beta}{d_{1}-d_{3}}+\frac{\alpha+\beta}{d_{2}-d_{3}}-\frac{q}{4 k^{2} d_{3}} \alpha \beta\right)=\frac{h}{4 k^{2}} \beta^{2}-4+\frac{\beta \alpha_{1}+\alpha \alpha_{3}}{k} .
\end{aligned}
$$

Consequently, the left-hand sides of the previous equations must be close to 0 if we want $\lambda$ to go to 0 . With the specific choice of charges and dipole charges (2.24), analytic solutions can be found

$$
\begin{aligned}
& \alpha=\frac{4 k^{2}}{q} \frac{a_{2}^{3} a_{3}+a_{1}^{2} a_{3}\left(2 a_{2}-a_{3}\right)+a_{1} a_{2}\left(a_{2}^{2}-5 a_{2} a_{3}+2 a_{3}^{2}\right)}{a_{2}\left(a_{1}-a_{2}\right)\left(a_{1}-a_{3}\right)\left(a_{2}-a_{3}\right)}+\mathcal{O}(\lambda) \\
& \beta=\frac{4 k^{2}}{q} \frac{a_{2}^{3} a_{3}+a_{1}^{2} a_{3}\left(2 a_{2}-a_{3}\right)+a_{1} a_{2}\left(a_{2}^{2}-5 a_{2} a_{3}+2 a_{3}^{2}\right)}{a_{1}\left(a_{1}-a_{2}\right)\left(a_{2}-a_{3}\right)^{2}}+\mathcal{O}(\lambda) \\
& \gamma=\frac{4 k^{2}}{q} \frac{a_{2}^{3} a_{3}+a_{1}^{2} a_{3}\left(2 a_{2}-a_{3}\right)+a_{1} a_{2}\left(a_{2}^{2}-5 a_{2} a_{3}+2 a_{3}^{2}\right)}{a_{3}\left(a_{1}-a_{2}\right)^{2}\left(a_{2}-a_{3}\right)}+\mathcal{O}(\lambda) .
\end{aligned}
$$

The main difference between our solutions and the solutions in [9] is that the three centers and the Gibbons-Hawking center are scaling together in our solutions, while in [9] only supertubes were scaling. This will prove to be valuable when we construct scaling solutions with four GH centers. Those formulas are useful to have an idea about the values of charges we need to choose to obtain a particular scaling solution with three supertubes in Taub-NUT. We apply those formulas in the next sections to find specific examples of solutions with no difference in scales between the inter-center distances.

\subsubsection{Solutions with $h=0$ and with no constant terms in all $K^{I}$}

Imposing $h=0$ and $q=1$ is principally motivated by having a Gibbons-Hawking metric which looks like $\mathbb{R}^{4}$ at infinity. The first example of charges and dipole charges is

$$
\begin{aligned}
h & =0 \\
q & =1 \\
k_{1} & =k_{3}=-k_{2}=k=100 \\
Q_{1}^{(2)} & =Q_{3}^{(2)}=\alpha=70000 \\
Q_{3}^{(1)} & =Q_{2}^{(3)}=\beta=100000 \\
Q_{2}^{(1)} & =Q_{1}^{(3)}=\gamma=233333 .
\end{aligned}
$$

We solve numerically the bubble equations (2.21):

$$
a_{1}=8.46360 \ldots \times 10^{-2}, \quad a_{2}=4.02091 \ldots \times 10^{-2}, \quad a_{3}=1.80731 \ldots \times 10^{-2} .
$$

One can check straightforwardly by plotting the quartic invariant $I_{4}$ and $\omega^{2}$ in the $\mathbb{R}^{3}$ space that the no-CTC condition (2.23) is satisfied.

We notice that many other different scaling solutions can be built from (2.23). 


\section{Charges, angular momentum and entropy of the solution}

We give also the charges and the angular momentum of the solution:

$$
\begin{aligned}
Q_{1} & =Q_{3}=\frac{293333}{4} \\
Q_{2} & =45000 \\
J & =\frac{44666625}{8} .
\end{aligned}
$$

The Bekenstein-Hawking entropy of the corresponding black hole is then

$$
S=2 \pi \sqrt{Q_{1} Q_{2} Q_{3}-J^{2}}=9.12309 \ldots \times 10^{7} .
$$

We compute the entropy parameter $\mathcal{H}$ which informs how far the charges of the solution are from those of the corresponding maximally-spinning black hole.

$$
\mathcal{H}=0.87 \ldots
$$

\section{Scaling solutions}

Finally, we scale the solution. We fine-tune the values of the initial charges, we solve the bubble equation, and then we check the absence of CTCs. The scaling process is summed up in the following table:

\begin{tabular}{|c|c|c|c|c|c|c|c|c|}
\hline Sol & $\mathrm{q}$ & $\mathrm{k}$ & $\alpha$ & $\beta$ & $\gamma$ & $a_{1}$ & $\frac{a_{1}}{a_{2}}$ & $\frac{a_{1}}{a_{3}}$ \\
\hline 1 & 1 & 100 & 70000 & 100000 & 233333 & $8.46360 \times 10^{-2}$ & 2.10490 & 4.68298 \\
\hline 2 & 1 & 100 & 70000 & 100000 & 233333,3 & $8.46360 \times 10^{-3}$ & 2.10490 & 4.68298 \\
\hline 3 & 1 & 100 & 70000 & 100000 & 233333,33 & $8.46361 \times 10^{-4}$ & 2.10489 & 4.68298 \\
\hline 4 & 1 & 100 & 70000 & 100000 & 233333,333 & $8.46360 \times 10^{-5}$ & 2.10490 & 4.68298 \\
\hline 5 & 1 & 100 & 70000 & 100000 & 233333,33333 & $8.46360 \times 10^{-7}$ & 2.10490 & 4.68298 \\
\hline
\end{tabular}

Therefore, when $\gamma$ is converging towards a certain value, the supertube positions shrink drastically. When the centers get closer and closer, the size of the throat gets larger and larger as explained in [7] and depicted in figure 1. Because a BPS black hole has an infinite throat, the scaling process makes the bubbling solution get more and more similar to a BPS black hole. We find a specific example of scaling microstate geometry of three BPS supertubes in $\mathbb{R}^{4}$ corresponding to a BMPV microstate with $\mathcal{H}=0.87$.

\subsubsection{Solutions with $h \neq 0$}

Adding a non-zero constant term in $V$ will make the Gibbons-Hawking metric look like $\mathbb{R}^{3} \times S^{1}$ at infinity. Such a configuration will be convenient when spectral flows and gauge transformations will be applied to reach general BPS solutions with four GH centers. To cope with the constant term in $V$, constant terms in $K^{I}$ must be turned on to satisfy the absence of the CTCs at infinity (2.23). We fix the constant terms as follows:

$$
\begin{aligned}
& \alpha_{1}=-2 h m_{\infty} \\
& \alpha_{2}=\alpha_{3}=0 .
\end{aligned}
$$



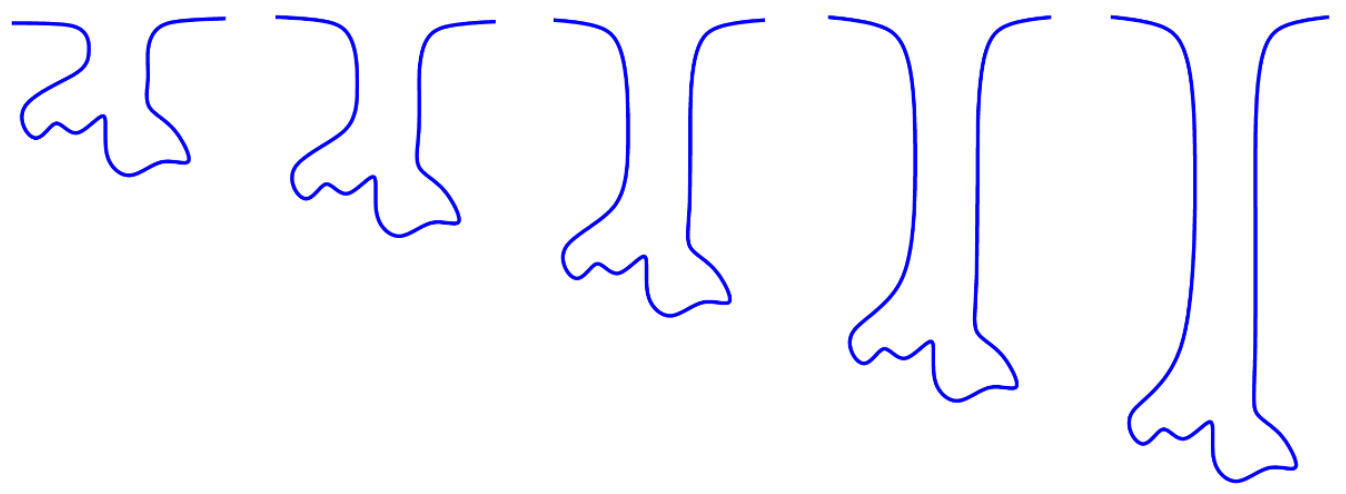

Figure 1. A schematic description of a scaling geometry. The physical size of the bubbles remains the same whereas the throat of the solutions deepens [7].

Indeed, such a choice ensures that

$$
\begin{aligned}
\mu(\infty) & =0 \\
Z_{I}(\infty) & =1 .
\end{aligned}
$$

We choose the following values of charges and dipole charges as an illustration:

$$
\begin{aligned}
h & =1 \\
q & =1 \\
k_{1} & =k_{3}=-k_{2}=k=100 \\
Q_{1}^{(2)} & =Q_{3}^{(2)}=\alpha=70000 \\
Q_{3}^{(1)} & =Q_{2}^{(3)}=\beta=100000 \\
Q_{2}^{(1)} & =Q_{1}^{(3)}=\gamma=240000 .
\end{aligned}
$$

The bubble equations (2.21) are solvable, the no-CTC condition is satisfied and the threecenter positions are

$$
a_{1}=3.04216 \ldots \times 10^{-2}, \quad a_{2}=1.50188 \ldots \times 10^{-2}, \quad a_{3}=6.69370 \ldots \times 10^{-3} .
$$

\section{Charges, angular momentum and entropy of the solution}

The charges and angular momentum of the solution are:

$$
\begin{aligned}
Q_{1} & =Q_{3}=75000 \\
Q_{2} & =45000 \\
J & =5687500 .
\end{aligned}
$$

The Bekenstein-Hawking entropy of the corresponding black hole solution is

$$
S=2 \pi \sqrt{Q_{1} Q_{2} Q_{3}-J^{2}}=9.33592 \ldots \times 10^{7} .
$$

Finally, the entropy parameter $\mathcal{H}$ is

$$
\mathcal{H}=0.87 \ldots
$$




\section{Scaling solutions}

As the previous section, we scale the solution by adjusting only the value of $\gamma$. The scaling process is summed up in the following table:

\begin{tabular}{|c|c|c|c|c|}
\hline Solutions & $\gamma$ & $a_{1}$ & $\frac{a_{1}}{a_{2}}$ & $\frac{a_{1}}{a_{3}}$ \\
\hline 1 & 240000 & $3.04216 \times 10^{-2}$ & 2.02557 & 4.54481 \\
\hline 2 & 234000 & $2.97358 \times 10^{-3}$ & 2.09593 & 4.66672 \\
\hline 3 & 233400 & $2.96688 \times 10^{-4}$ & 2.10405 & 4.68150 \\
\hline 4 & 233340 & $2.96621 \times 10^{-5}$ & 2.10481 & 4.68283 \\
\hline 5 & 233333,4 & $2.96614 \times 10^{-7}$ & 2.10490 & 4.68299 \\
\hline
\end{tabular}

We find an example of scaling microstate geometry of three BPS supertubes with one Gibbons-Hawking center in Taub-NUT. Furthermore, if we compare both tables with $h=1$ and with $h=0$, the solutions are really similar to each other in the scaling limit. So the constant terms of the harmonic functions are irrelevant in the scaling limit.

\section{Constructing BPS solutions with four Gibbons-Hawking centers}

\subsection{Four-GH center bubbled solutions}

In this section, we consider BPS bubbled solutions with four GH centers. Those solutions can be entirely generated from the family of three-supertube solutions by using three generalized spectral flows and three gauge transformations. The eleven-dimensional metric is the same as (2.1). However, we consider a much more general set of harmonic functions $\left\{V, K^{I}, L_{I}, M\right\}$. Each function has poles at each GH center. We use the following usual notations:

$$
\begin{gathered}
V=q_{\infty}+\sum_{j=0}^{3} \frac{q_{j}}{r_{j}}, \quad M=m_{\infty}+\sum_{j=0}^{3} \frac{m_{j}}{r_{j}} \\
K^{I}=k_{\infty}^{I}+\sum_{j=0}^{3} \frac{k_{j}^{I}}{r_{j}}, \quad L_{I}=l_{\infty}^{I}+\sum_{j=0}^{3} \frac{l_{j}^{I}}{r_{j}},
\end{gathered}
$$

$r_{0}, r_{1}, r_{2}$ and $r_{3}$ are the same distances as in the first section with $r_{0}$ corresponding to $r$. In this framework, the regularity constraints are

$$
\begin{array}{cc}
l_{j}^{I}=-\frac{1}{2} C_{I J K} \frac{k_{j}^{J} k_{j}^{K}}{q_{j}} & j \in\{0, \ldots, 3\} \\
m_{j}=\frac{1}{12} C_{I J K} \frac{k_{j}^{I} k_{j}^{J} k_{j}^{K}}{q_{j}^{2}} & j \in\{0, \ldots, 3\} .
\end{array}
$$

The equations (2.10) for the warp factors $Z_{I},(2.12)$ for $\mu,(2.14)$ for $\omega$, the bubble equations (2.18) and the no-CTC condition (2.23) are still valid. 


\subsection{Spectral flows and gauge transformations}

For BPS solutions, generalized spectral flows and gauge transformations are wellunderstood $[19,20]$. They mix the harmonic functions leaving the bubble equations and the entropy function invariant. Furthermore, they send the family of three-supertube solutions to the family of solutions with four GH centers. The harmonic functions $\left\{V, K^{I}, L_{i}, M\right\}$ of BPS solutions transform under three gauge transformations parameterized by three constants $g^{I}$ as [21]:

$$
\begin{aligned}
& V \rightarrow V, \quad K^{I} \rightarrow K^{I}+g^{I} V, \\
& L^{I} \rightarrow L^{I}-C_{I J K} g^{J} K^{K}-\frac{1}{2} C_{I J K} g^{J} g^{K} V, \\
& M \rightarrow M-\frac{1}{2} g^{I} L^{I}+\frac{1}{4} C_{I J K} g^{I} g^{J} K^{K}+\frac{1}{12} C_{I J K} g^{I} g^{J} g^{K} V,
\end{aligned}
$$

and under a family of generalized spectral flows parameterized by three constants $\gamma^{I}$ as [19]:

$$
\begin{aligned}
& M \rightarrow M, \\
& K^{I} \rightarrow L^{I}-2 \gamma^{I} M, \\
& \rightarrow K^{I}-C_{I J K} \gamma^{J} L^{K}+C_{I J K} \gamma^{J} \gamma^{K} M, \\
& V \rightarrow V+\gamma^{I} K^{I}-\frac{1}{2} C_{I J K} \gamma^{I} \gamma^{J} L^{K}+\frac{1}{3} C_{I J K} \gamma^{I} \gamma^{J} \gamma^{K} M .
\end{aligned}
$$

\subsection{One particular example of solutions}

We start from the particular solution of three supertubes in Taub-NUT background of section 2.3.3 with $h \neq 0$ and $\gamma=233400$. We perform three independent spectral flows then three independent gauge transformations. To obtain physical solutions and a GibbonsHawking metric which looks like $\mathbb{R}^{4}$ at infinity, the transformations are constrained such that in the resulting solution:

- The constant term in $V$ is 0 .

- The sum of all charges in $V$ is 1 .

- The charges in $V$ are integers.

- The constant terms in all $K^{I}$ are 0 .

- The values of the charges and dipole charges are rational [22].

To satisfy the conditions above, we first leave a non-zero constant term in $V$ in order to remove the constant terms in each $K^{I}$ with three gauge transformations. Then we fix one spectral flow parameter to satisfy the second condition. The third condition cannot be easily satisfied. So, we fix the two remaining spectral flow parameters to have the closest values to integers. Afterwards, all six transformation parameters are fixed. We remove by hand the constant term in $V$. Because the bubble equations are changing significantly, we have to solve them accordingly. For this purpose, two options exist: 
- We solve the bubble equations by considering the positions of the centers as variables and all the charges are fixed.

- We solve the bubble equation by considering the charges as variables and the positions are fixed. In the scaling limit, one can see straightforwardly that an infinitesimal change of three dipole charges $\left(k_{0}^{1}, k_{0}^{2}\right.$ and $k_{0}^{3}$ for instance) can annihilate a change of order one on the right hand side of the bubble equations (2.18). Consequently, one can find a solution to the bubble equations only perturbing infinitesimally the values of the charges which ensures that no closed timelike curves will appear.

In general, we apply the first method. However, when it was not possible to do, we apply the second one.

At that point, the values of charges and dipole charges are real numbers with an infinite number of digits. The last step is to round the values taking into account the necessary regularity conditions (3.2). Once again, the solutions are not invariant, we have to check that the bubble equations are still solvable and that the no-CTC condition is still satisfied.

We present the result of applying the above procedures on the three-supertube configuration with $h \neq 0$ and $\gamma=233400$ :

$$
\begin{aligned}
V= & \frac{1}{r}+\frac{1}{r_{1}}+\frac{12}{r_{2}}-\frac{13}{r_{3}} \\
K^{1}= & -\frac{2087}{10000} \frac{1}{r}-\frac{678089}{1250} \frac{1}{r_{1}}+\frac{55636379}{10000} \frac{1}{r_{2}}+\frac{3445309}{2000} \frac{1}{r_{3}} \\
K^{2}= & -\frac{491}{2500} \frac{1}{r}+\frac{4712993}{1250} \frac{1}{r_{1}}+\frac{30306499}{5000} \frac{1}{r_{2}}+\frac{32175101}{5000} \frac{1}{r_{3}} \\
K^{3}= & \frac{1}{10000} \frac{1}{r}-\frac{49939}{10000} \frac{1}{r_{1}}-\frac{311181}{5000} \frac{1}{r_{2}}+\frac{133657}{2000} \frac{1}{r_{3}} \\
L^{1}= & 1+\frac{491}{25000000} \frac{1}{r}+\frac{235362157427}{12500000} \frac{1}{r_{1}}+\frac{3143602221773}{100000000} \frac{1}{r_{2}}+\frac{4300427474357}{130000000} \frac{1}{r_{3}} \\
L^{2}= & 1+\frac{2087}{100000000} \frac{1}{r}-\frac{33863086571}{12500000} \frac{1}{r_{1}}+\frac{5770994684533}{200000000} \frac{1}{r_{2}}+\frac{460489665013}{52000000} \frac{1}{r_{3}} \\
L^{3}= & 1-\frac{1024717}{25000000} \frac{1}{r}+\frac{3195828710377}{1562500} \frac{1}{r_{1}}-\frac{1686143864527121}{600000000} \frac{1}{r_{2}} \\
& +\frac{110853165051209}{130000000} \frac{1}{r_{3}} \\
M= & -\frac{115048645}{10000}-\frac{1024717}{500000000000} \frac{1}{r}+\frac{159596489967517003}{31250000000} \frac{1}{r_{1}} \\
& -\frac{174898644635804679967}{24000000000000} \frac{1}{r_{2}}+\frac{14816301481249441313}{6760000000000} \frac{1}{r_{3}} .
\end{aligned}
$$

The bubble equations are solved by the following positions:

$$
a_{1}=2.67495 \ldots \times 10^{-2}, \quad a_{2}=1.27076 \ldots \times 10^{-2}, \quad a_{3}=5.70977 \ldots \times 10^{-3} .
$$




\section{Charges, angular momentum and entropy}

The charges and angular momentum of the solution are:

$$
\begin{aligned}
Q_{1} & =\frac{99858458954459}{1300000000} \\
Q_{2} & =\frac{83964235108323}{2600000000} \\
Q_{3} & =\frac{856306630373655247}{7800000000} \\
J & =\frac{705272590995929898902049}{1352000000000000} .
\end{aligned}
$$

The Bekenstein-Hawking entropy of the corresponding black hole solution is

$$
S=9.12620 \ldots \times 10^{7} .
$$

Finally, the entropy parameter $\mathcal{H}$ is

$$
\mathcal{H}=0.000775 .
$$

The angular momentum is extremely close to the maximally spinning value. This is not a coincidence and this is one of the key points of our paper. Picking random three-supertube configuration also gives a near-maximally spinning solution after spectral flows and gauge transformations. In order to prevent this, a very fine tuning between the parameters of the three-supertube solution is required. The main reason is that the charges $Q_{1}, Q_{2}$ and $Q_{3}$ strongly increase under spectral flows if all the conditions imposed for $V$ are satisfied. Consequently, the angular momentum is forced to reach a value close to $\sqrt{Q_{1} Q_{2} Q_{3}}$ to leave the entropy function invariant. This will be fully described in section 4 .

\subsubsection{Scaling solutions}

We check whether the new solution can still scale. We only change $k_{2}^{1}$, but any other $K^{I}$ coefficient would have worked. Furthermore, we take into account that any change of $k_{2}^{1}$ produces a change in the $L^{I}$ 's charges and in $\mathrm{M}$ according to (3.2). We give the steps in the following table:

\begin{tabular}{|c|c|c|c|c|}
\hline Sol & $k_{2}^{1}$ & $a_{1}$ & $\frac{a_{1}}{a_{2}}$ & $\frac{a_{1}}{a_{3}}$ \\
\hline 1 & 5563.6379 & $2.67494 \times 10^{-2}$ & 2.10499 & 4.68485 \\
\hline 2 & 5562.9979 & $5.47309 \times 10^{-3}$ & 2.10489 & 4.68358 \\
\hline 3 & 5562.8379 & $1.52860 \times 10^{-4}$ & 2.10487 & 4.68327 \\
\hline 4 & 5562.83334 & $1.22654 \times 10^{-6}$ & 2.10486 & 4.68326 \\
\hline 5 & 5562.822208 & $1.62447 \times 10^{-7}$ & 2.10487 & 4.68325 \\
\hline 6 & 5562.8333032 & $2.83275 \times 10^{-9}$ & 2.10487 & 4.68325 \\
\hline 7 & 5562.8333031 & $1.72517 \times 10^{-10}$ & 2.10486 & 4.68324 \\
\hline
\end{tabular}

Thus, we built asymptotically $\mathbb{R}^{4}$ solutions with a shrinking cluster of four bubbled GH centers with no difference in scales. These are microstates of a black hole with a macroscopically large horizon. In the next section we investigate how systematic the large $J$ is. 


\section{Discussion}

\subsection{Solutions with four GH centers with no difference in scales are necessarily near-maximally spinning}

Understanding the impact of spectral flows on a cluster of three supertubes in TaubNUT by an analytical approach is complicated due to the number of parameters and the complexity of the equations. However, by a numerical approach, it appears that performing three constrained spectral flows on a three-supertube solution with no difference in scales between the inter-center distances (that is to say $a_{3}, a_{2}-a_{3}$ and $a_{1}-a_{2}$ are of the same order of magnitude) will result in an entropy parameter extremely close to 0. By "constrained spectral flows" one means that the resulting harmonic function $V$ must have integer charges whose sum is equal to one. We reached this conclusion after having studied many different examples which have led to a value of $\mathcal{H}$ around 0.01 each time. Insights of our approach are given in the next two sections and in appendix A.

\subsubsection{Numerical analysis}

First, we investigate all the solutions with four GH centers where the centers scale as follows:

$$
\begin{aligned}
& \frac{a_{1}-a_{2}}{a_{3}} \approx 1 \\
& \frac{a_{2}-a_{3}}{a_{3}} \approx 1 .
\end{aligned}
$$

We would like to scan all the solutions of this kind, to compute their entropy parameters, and to find out if they are indeed all of the order of 0.01 .

Let us list all the free parameters. The starting configuration is a three-supertube solution with six positive charges $Q_{I}^{(J)}$, three dipole charges $k_{I}$ and one Gibbons-Hawking charge $q$. The constant terms do not enter in the formula for the entropy parameter and hence are irrelevant. The scaling conditions fix three charges. Furthermore, we notice that changes of $q$ can be reabsorbed into changes of dipole charges. So without loss of generality we can consider that $q$ is equal to one. We can also restrict the sign changes of dipole charges by symmetry. Then, all six parameters of the spectral flows and the gauge transformations are constrained to cancel the constant terms in all $K^{I}$ and to have a Gibbons-Hawking harmonic function of the form:

$$
V=\frac{1}{r}-\frac{2}{r_{1}}+\frac{1}{r_{2}}+\frac{1}{r_{3}}
$$

This constitutes the best choice to maximize the value of the entropy parameter as shown at the end of appendix A. The process to obtain such solutions is the same as the one given in section 3.3. Finally, one needs to vary only three charge ratios, the norms and some signs of the dipole charges to scan all the solutions satisfying (4.1) and (4.2). We built a huge number of such four-center solutions using a loop algorithm. We have computed their entropy parameter to establish the variation of the entropy parameter as a function of the free parameters. We concluded that in any situation the entropy parameter is at 
most of order of 0.01. Then, we extended to solutions which do not necessarily satisfy (4.1) and (4.2). We observed the same feature. All the details are presented in appendix A.

\subsubsection{An analytical argument}

We investigate the near-maximally spinning feature in the context of the specific choice of charges and dipole charges made in 2.3.1. With this specific choice it is easier to understand analytically why the conditions imposed to the spectral flows produce an important increase in the charges $Q_{1}, Q_{2}$ and $Q_{3}$ which requires an increase in $J$ to leave the entropy invariant. We sum up how we proceed in the following steps:

- We express the charges $\alpha, \beta$ and $\gamma$ using the scaling condition relation (2.28) to reduce the number of independent parameters. We obtain approximations as $F\left(\frac{a_{1}}{a_{3}}, \frac{a_{2}}{a_{3}}\right) \frac{4 k^{2}}{q}$ for each charge where the $F$ 's are rational fractions:

$$
\begin{array}{llll}
\alpha \approx \frac{4 k^{2}}{q} F_{\alpha}\left(\frac{a_{1}}{a_{3}}, \frac{a_{2}}{a_{3}}\right), & F_{\alpha}(x, y)= & \frac{y^{3}+x^{2}(2 y-1)+x y\left(y^{2}-5 y+2\right)}{y(x-y)(x-1)(y-1)} \\
\beta \approx \frac{4 k^{2}}{q} F_{\beta}\left(\frac{a_{1}}{a_{3}}, \frac{a_{2}}{a_{3}}\right), & F_{\beta}(x, y)= & \frac{y^{3}+x^{2}(2 y-1)+x y\left(y^{2}-5 y+2\right)}{x(x-y)(y-1)^{2}} \\
\gamma \approx \frac{4 k^{2}}{q} F_{\gamma}\left(\frac{a_{1}}{a_{3}}, \frac{a_{2}}{a_{3}}\right), & F_{\gamma}(x, y)= & \frac{y^{3}+x^{2}(2 y-1)+x y\left(y^{2}-5 y+2\right)}{(x-y)^{2}(y-1)} .
\end{array}
$$

- We analytically express how $Q_{1}, Q_{2}$ and $Q_{3}$ transform under three generalized spectral flows one of which is fixed by requiring the sum of the charges in $V$ to be equal to one. The expressions are too complicated to be written down but the main point is that the charges can be fully expressed according to 6 initial parameters $Q_{I}\left(k, q, \frac{a_{1}}{a_{3}}, \frac{a_{2}}{a_{3}}, \gamma^{2}, \gamma^{3}\right)$ where the $\gamma^{I}$ are the three spectral flow parameters with $\gamma^{1}$ fixed to have the sum of the charges in $V$ equal to 1 .

- We assume no difference in scale: $1.2 a_{3}<1.1 a_{2}<a_{1}<10 a_{3}$. Using numerics to perform a functional analysis on the charges $Q_{I}$ in those domains, we saw that $Q_{1}$, $Q_{2}$ and $Q_{3}$ do not double after spectral flows if the spectral flow parameters are at most of order $\frac{k}{q \alpha} \sim \frac{1}{4 F k}$.

- Finally, by studying the transformation of $V$ under spectral flows in the specific domains of parameters found above, one proves that the charges in $V$ induced by the spectral flow parameters are smaller than one which is non-physical. This can be easily intuited looking at each term of $V \rightarrow V+\gamma^{I} K^{I}-\frac{1}{2} C_{I J K} \gamma^{I} \gamma^{J} L^{K}+$ $\frac{1}{3} C_{I J K} \gamma^{I} \gamma^{J} \gamma^{K} M$. If we replace the spectral flow parameters with $\frac{1}{4 F k}$, the orders of magnitude of each term are straightforwardly below one (we remind that $q>1$ in order to have $q_{0}>1$, the final charge of $V$ at the first center).

This technical argument conveys the idea that in the domains of parameters where the distances between the center do not have difference in scale, the spectral flow parameters are significantly high to produce integer-value charges in $V$ and they are too high to avoid the charges $Q_{I}$ not to increase too much. This should come from the structure of the 
variations of the charges and angular momentum under spectral flows which are hard to study without numerics.

With those two analyses, we conclude that any BPS bubbled scaling solution with four GH centers with no difference in scales is necessarily near-maximally spinning.

By the same kind of arguments, if no assumption about the positions is made, building a solution which is not a near-maximally spinning solution is also difficult but not impossible [12].

\section{Conclusion}

We have built several examples of four-center BPS bubbled solutions. Starting with three supertubes in Taub-NUT, we gave a systematic protocol and we built several scaling solutions. Secondly, using generalized spectral flows we generated BPS solutions with four Gibbons-Hawking centers. We gave a numerical protocol to obtain such solutions from three-supertube scaling solutions and we built specific examples. We reached one important conclusion: BPS solutions with four GH centers that do not have an entropy parameter close to 0 are rare. Furthermore, when such solutions have no difference in scales between the inter-center distances, they are necessarily near-maximally spinning.

There are several interesting directions for future research. The first is to find BPS solutions with four GH centers which are not near-maximally spinning. We expect them to display a difference in scales between the inter-center distances, as in the examples built in [7]. Using algorithms to fine-tune the initial three-supertube parameters, one can plan to find such solutions [12]. One can also investigate different four-center constructions with one supertube and three Gibbons-Hawking centers. Those solutions are smooth in the D1D5-P duality frame and can be generated from three-supertube solutions by two spectral flows. Thus, one may expect them not to necessarily be near-maximally spinning solutions even when the centers have no difference in scale. Secondly, one can extend our work to almost-BPS four-center solutions [23] following the same ideas. Thirdly one can add nonAbelian hair to the solutions as it was done in [24]. Finally, one can also investigate whether solutions with more than four Gibbons-Hawking centers without difference in scale are also near-maximally spinning and, more generally, how universal is the feature we found.

\section{Acknowledgments}

I would like to thank Iosif Bena and Pedro Ramirez for the interesting discussions. This work was supported by an ENS Lyon doctoral grant.

\section{A Numerical analysis of the entropy parameter of four-GH center solu- tions}

In this section, we detail how we scanned all the different values of the entropy parameter we can get from scaling solutions with four Gibbons-Hawking centers. As explained in 
section 4.1.1, we first scanned all the scaling solutions which satisfy

$$
\begin{aligned}
& \frac{a_{1}-a_{2}}{a_{3}} \approx 1 \\
& \frac{a_{2}-a_{3}}{a_{3}} \approx 1 \\
& V=\frac{1}{r}-\frac{2}{r_{1}}+\frac{1}{r_{2}}+\frac{1}{r_{3}},
\end{aligned}
$$

by varying the free parameters of the constructions: three charge ratios $\left(\frac{Q_{2}^{(1)}}{Q_{1}^{(3)}}, \frac{Q_{3}^{(2)}}{Q_{1}^{(2)}}\right.$ and $\frac{Q_{2}^{(3)}}{Q_{3}^{(1)}}$ for instance) and the three dipole charges $k_{1}, k_{2}$ and $k_{3}$. The graphs figure 2 give the variations of the entropy parameter according to three charge ratios from 0.1 to 4 with $q, k_{1},-k_{2}$ and $k_{3}$ equal to 1 . Each graph has been built with 10000 scaling solutions which are not necessarily CTC-free and the result has been interpolated and smoothed for readability. Thus, it is important to point out that a point of one curve does not necessarily correspond to a CTC-free solution with four GH centers. Nevertheless, any CTC-free fourcenter solution meeting the conditions above has an entropy parameter near or below the curve's prediction.

In the domains studied, the entropy parameter is indeed of order 0.01. One also has to check the regions where $\mathcal{H}$ increases. For this purpose, we have investigated the evolution of the entropy parameter as a function of one of the charge ratios for a larger range of values. We observed that the evolutions for any value of the two other charge ratios are similar to the four evolutions shown in figure 3 and that $\mathcal{H}$ is bounded between 0 and 0.09 . However it is important to point out that in the domains of very large or very small charge ratios the solutions can possibly have closed timelike curves. Indeed, if we want the four centers to scale in one block, the bubble equations (2.18) imply that one supertube at least must have a negative dipole charge. Consequently, if some charges are small compared to the value of the dipole charge, the warp factor (2.10) of the corresponding supertube may be negative around other poles. Thus, the upper bound 0.09 of the entropy parameter can be thought of as a generous upper bound for CTC-free solutions.

Similarily, we studied the entropy parameter as a function of the dipole charges and their signs. We found in all configurations the same kind of evolutions as in figure 2. Consequently, any entropy parameter of solutions with four Gibbons-Hawking centers satisfying (4.1) and (4.2) are of the order of 0.01. The last step is to ensure that solutions which do not satisfy (4.1) and (4.2) have the same property. The graph figure 4 illustrates that if the GH function $V$ has higher charges the entropy parameter is getting closer to 0 . So the condition (4.2) gives the solutions which have the highest values of $\mathcal{H}$. We vary also the condition (4.1) taking into account that the inter-center distances must remain of the same order. The graphs figure 5 show that changing the aspect ratio $\frac{a_{1}}{a_{2}}$ does not produce a significant change of the entropy parameter.

This exhaustive numerical analysis shows that any solution with four GH centers with no difference in scales is necessarily near-maximally spinning. 


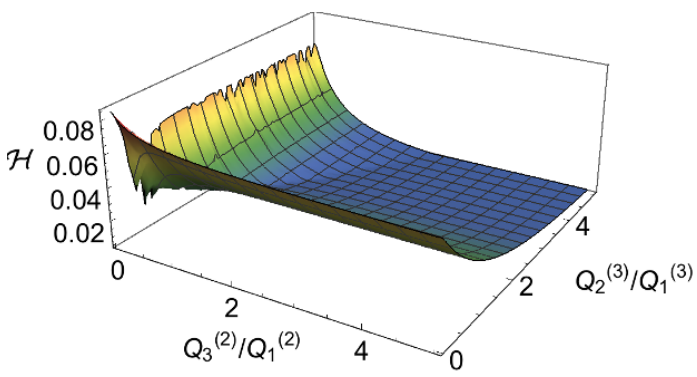

(a) $\frac{Q_{2}^{(1)}}{Q_{1}^{(3)}}=0.1$

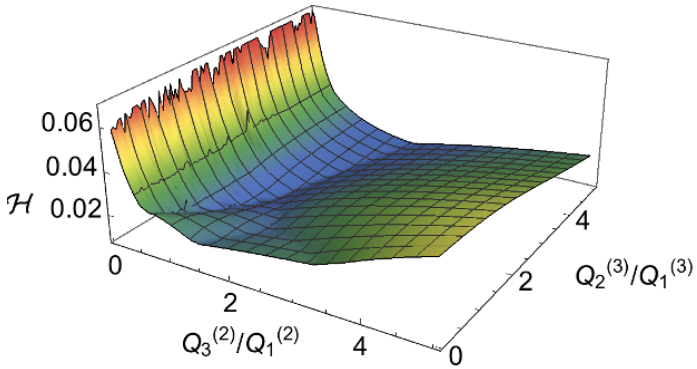

(c) $\frac{Q_{2}^{(1)}}{Q_{1}^{(3)}}=1$

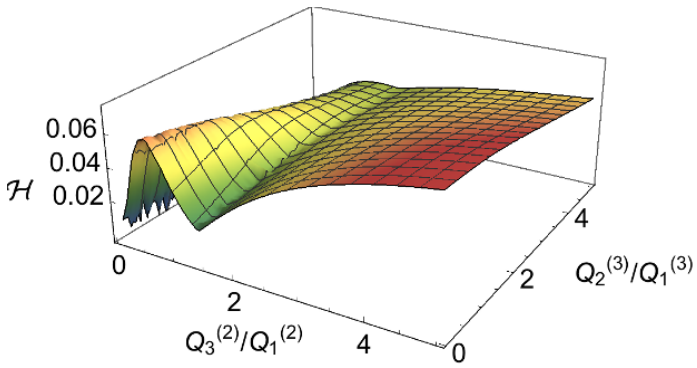

(e) $\frac{Q_{2}^{(1)}}{Q_{1}^{(3)}}=5$

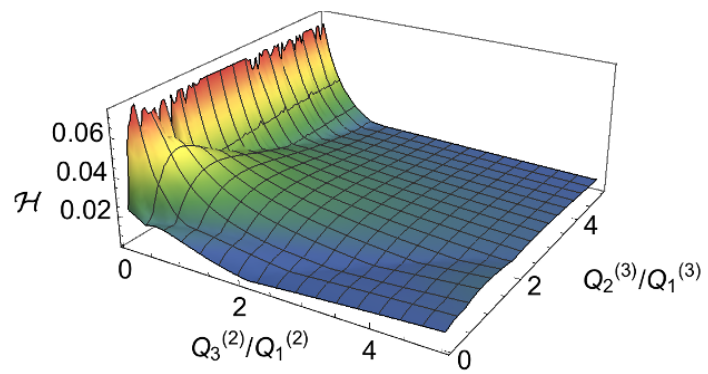

(b) $\frac{Q_{2}^{(1)}}{Q_{1}^{(3)}}=0.5$

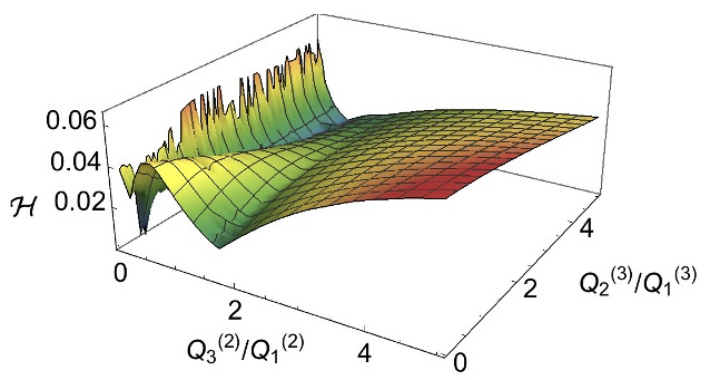

(d) $\frac{Q_{2}^{(1)}}{Q_{1}^{(3)}}=2$

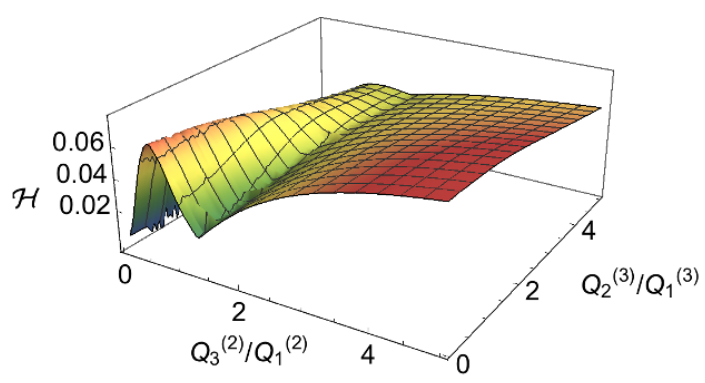

(f) $\frac{Q_{2}^{(1)}}{Q_{1}^{(3)}}=10$

Figure 2. The entropy parameter $\mathcal{H}$ as a function of the charge ratios with $q, k_{1},-k_{2}$ and $k_{3}$ equal to 1 . 


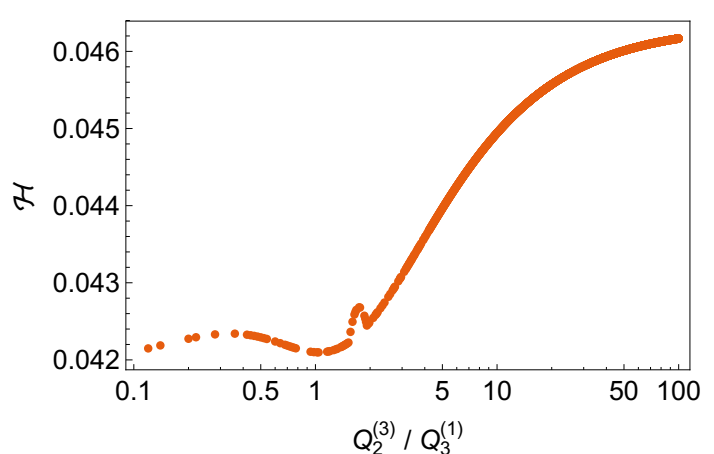

(a) $\frac{Q_{2}^{(1)}}{Q_{1}^{(3)}}=1$ and $\frac{Q_{3}^{(2)}}{Q_{1}^{(2)}}=0.2$

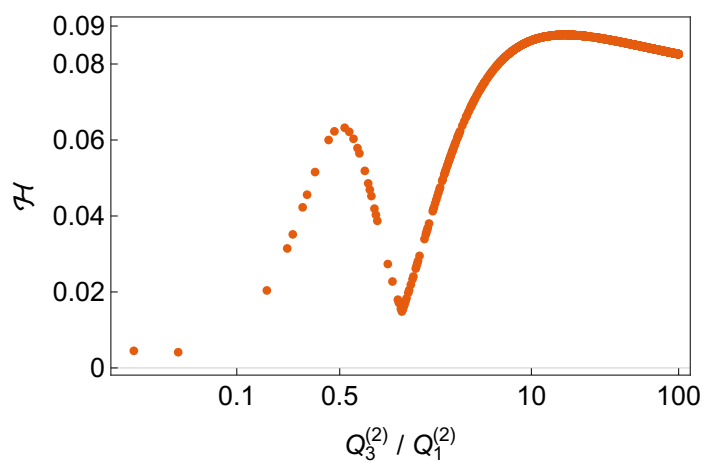

(c) $\frac{Q_{2}^{(1)}}{Q_{1}^{(3)}}=7$ and $\frac{Q_{2}^{(3)}}{Q_{3}^{(1)}}=0.003$

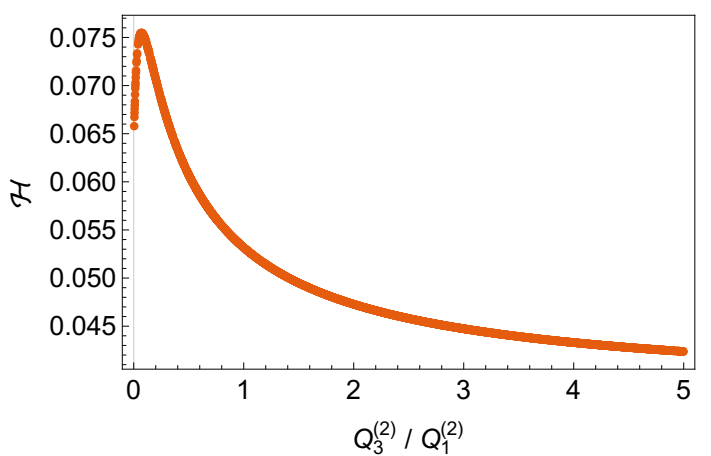

(b) $\frac{Q_{2}^{(1)}}{Q_{1}^{(3)}}=0.2$ and $\frac{Q_{2}^{(3)}}{Q_{3}^{(1)}}=0.2$

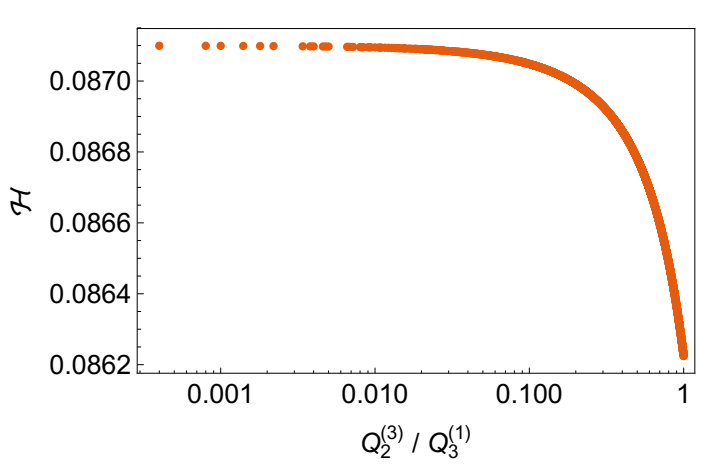

(d) $\frac{Q_{2}^{(1)}}{Q_{1}^{(3)}}=7$ and $\frac{Q_{3}^{(2)}}{Q_{1}^{(2)}}=12$

Figure 3. The entropy parameter $\mathcal{H}$ as a function of one of the charge ratios with $q, k_{1},-k_{2}$ and $k_{3}$ equal to 1 .

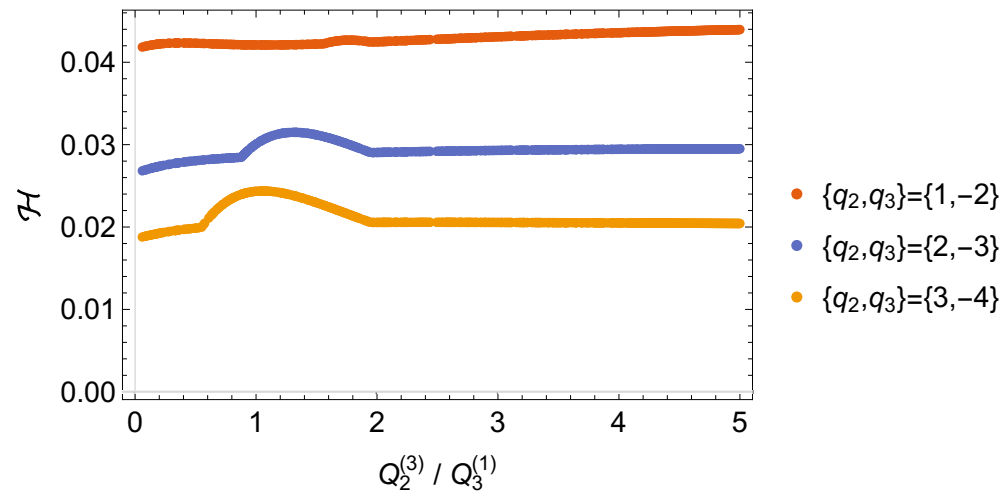

Figure 4. The entropy parameter $\mathcal{H}$ as a function of one of the charge ratios for three different GH functions $V$ with $q=1, k_{1}=-k_{2}=k_{3}=1, \frac{Q_{2}^{(1)}}{Q_{1}^{(3)}}=1$ and $\frac{Q_{3}^{(2)}}{Q_{1}^{(2)}}=0.2$. 


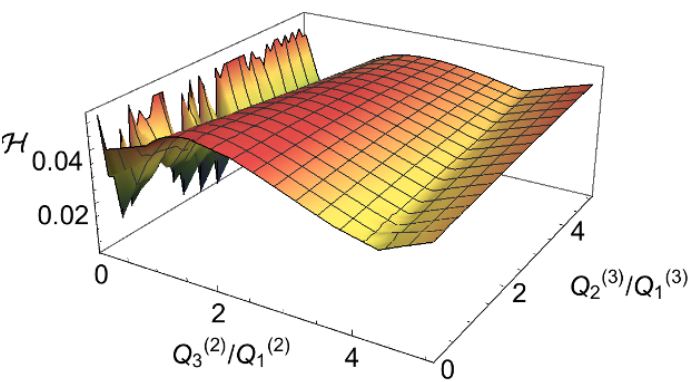

(a) $\frac{a_{1}}{a_{2}} \approx 1.1$

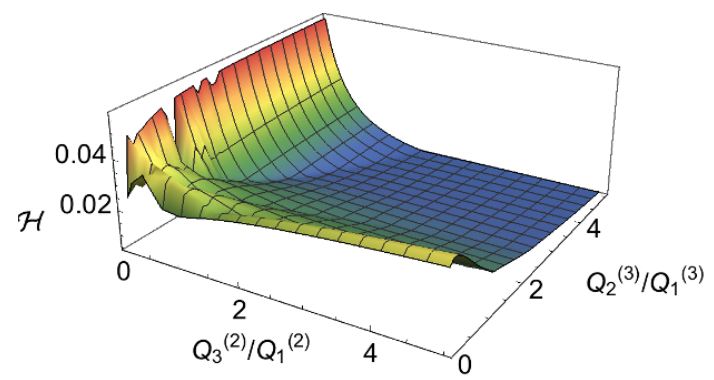

(c) $\frac{a_{1}}{a_{2}} \approx 5.6$

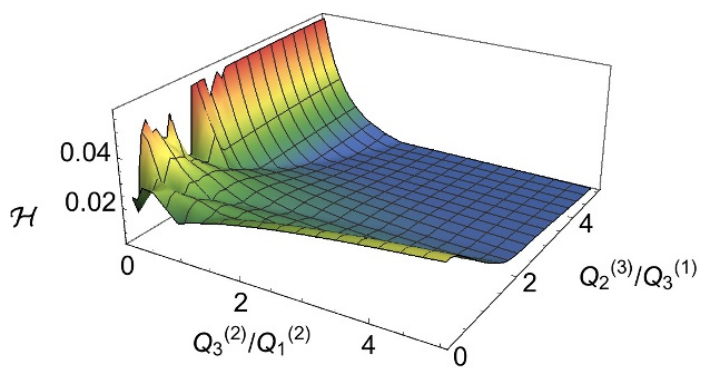

(b) $\frac{a_{1}}{a_{2}} \approx 3.1$

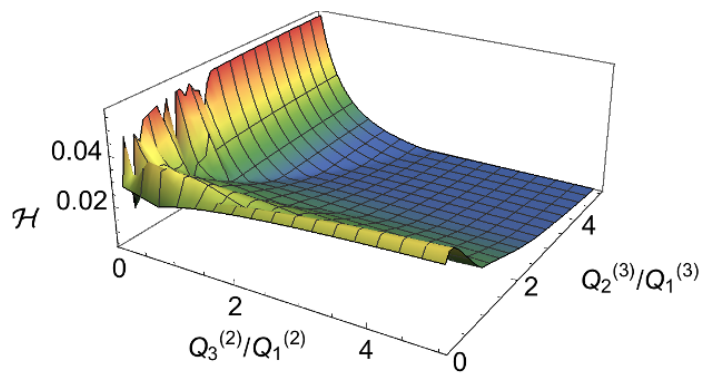

(d) $\frac{a_{1}}{a_{2}} \approx 10.1$

Figure 5. The entropy parameter $\mathcal{H}$ as a function of two charge ratios and one distance ratio with $q, k_{1},-k_{2}$ and $k_{3}$ equal to 1 .

Open Access. This article is distributed under the terms of the Creative Commons Attribution License (CC-BY 4.0), which permits any use, distribution and reproduction in any medium, provided the original author(s) and source are credited.

\section{References}

[1] S.D. Mathur, The fuzzball proposal for black holes: an elementary review, Fortsch. Phys. 53 (2005) 793 [hep-th/0502050] [INSPIRE].

[2] I. Bena and N.P. Warner, Black holes, black rings and their microstates, Lect. Notes Phys. 755 (2008) 1 [hep-th/0701216] [INSPIRE].

[3] S.D. Mathur, Fuzzballs and the information paradox: A Summary and conjectures, arXiv:0810.4525 [INSPIRE].

[4] V. Balasubramanian, J. de Boer, S. El-Showk and I. Messamah, Black holes as effective geometries, Class. Quant. Grav. 25 (2008) 214004 [arXiv:0811.0263] [InSPIRE].

[5] I. Bena and N.P. Warner, Bubbling supertubes and foaming black holes, Phys. Rev. D 74 (2006) 066001 [hep-th/0505166] [INSPIRE].

[6] F. Denef, Supergravity flows and D-brane stability, JHEP 08 (2000) 050 [hep-th/0005049] [INSPIRE].

[7] I. Bena, C.-W. Wang and N.P. Warner, Mergers and typical black hole microstates, JHEP 11 (2006) 042 [hep-th/0608217] [INSPIRE]. 
[8] I. Bena, C.-W. Wang and N.P. Warner, Plumbing the abyss: black ring microstates, JHEP 07 (2008) 019 [arXiv: 0706.3786] [INSPIRE].

[9] O. Vasilakis and N.P. Warner, Mind the gap: supersymmetry breaking in scaling, microstate geometries, JHEP 10 (2011) 006 [arXiv:1104.2641] [INSPIRE].

[10] M. Bianchi, J.F. Morales, L. Pieri and N. Zinnato, More on microstate geometries of 4 d black holes, JHEP 05 (2017) 147 [arXiv:1701.05520] [INSPIRE].

[11] I. Bena, C.-W. Wang and N.P. Warner, The foaming three-charge black hole, Phys. Rev. D 75 (2007) 124026 [hep-th/0604110] [INSPIRE].

[12] P. Heidmann and P. Ramirez, A systematic construction of microstate geometries with low angular momentum, arXiv: 1709.02812 [INSPIRE].

[13] I. Bena, S. Giusto, R. Russo, M. Shigemori and N.P. Warner, Habemus superstratum! A constructive proof of the existence of superstrata, JHEP 05 (2015) 110 [arXiv:1503.01463] [INSPIRE].

[14] I. Bena et al., Smooth horizonless geometries deep inside the black-hole regime, Phys. Rev. Lett. 117 (2016) 201601 [arXiv: 1607.03908] [INSPIRE].

[15] I. Bena, S. Giusto, C. Ruef and N.P. Warner, Multi-center non-BPS black holes: the solution, JHEP 11 (2009) 032 [arXiv: 0908.2121] [INSPIRE].

[16] J.B. Gutowski and H.S. Reall, General supersymmetric AdS $S_{5}$ black holes, JHEP 04 (2004) 048 [hep-th/0401129] [INSPIRE].

[17] I. Bena and N.P. Warner, One ring to rule them all... and in the darkness bind them?, Adv. Theor. Math. Phys. 9 (2005) 667 [hep-th/0408106] [InSPIRE].

[18] P. Berglund, E.G. Gimon and T.S. Levi, Supergravity microstates for BPS black holes and black rings, JHEP 06 (2006) 007 [hep-th/0505167] [INSPIRE].

[19] I. Bena, N. Bobev and N.P. Warner, Spectral flow and the spectrum of multi-center solutions, Phys. Rev. D 77 (2008) 125025 [arXiv:0803.1203] [INSPIRE].

[20] G. Dall'Agata, S. Giusto and C. Ruef, U-duality and non-BPS solutions, JHEP 02 (2011) 074 [arXiv: 1012.4803] [INSPIRE].

[21] I. Bena, P. Kraus and N.P. Warner, Black rings in Taub-NUT, Phys. Rev. D 72 (2005) 084019 [hep-th/0504142] [INSPIRE].

[22] V. Balasubramanian, E.G. Gimon and T.S. Levi, Four dimensional black hole microstates: from D-branes to spacetime foam, JHEP 01 (2008) 056 [hep-th/0606118] [INSPIRE].

[23] K. Goldstein and S. Katmadas, Almost BPS black holes, JHEP 05 (2009) 058 [arXiv: 0812.4183] [INSPIRE].

[24] P.F. Ramirez, Non-Abelian bubbles in microstate geometries, JHEP 11 (2016) 152 [arXiv:1608.01330] [INSPIRE]. 